\title{
SSinteza
}

Impact of Internet on Business Activities in Serbia and Worldwide

Uticaj Interneta na poslovanje u Srbiji i svetu

DOI: 10.15308/SINTEZA-2014-948-952

\section{PRIMENA TF/SF EKSITACIJE KOD RAČUNARSKIH FDTD SIMULACIJA ELEKTROMAGNETSKIH POLJA}

\author{
Branko D. Gvozdić, Dušan Ž. Đurđević \\ Univerzitet u Prištini sa sedištem u Kosovskoj Mitrovici, \\ Fakultet tehničkih nauka, Kosovska Mitrovica, Republika Srbija
}

\begin{abstract}
:
U FDTD (Finite Difference Time Domain) računarskim simulacijama elektromagnetskih polja često je potrebno pored tačkastih izvora, čije se polje prostire u svim pravcima numeričkog domena, implementirati i izvore čije se polje prostire samo u jednom pravcu. To se može postići korišćenjem eksitacije polja u vidu TF/SF (Total Field/Scattered Field) incidentnih ravnih talasa. U radu je prikazana primena TF/SF ravnih talasa, koji u osnovi imaju sinusoidni i Rikerov impuls, a kod 2D FDTD simulacija polja. Prezentovani su numerički rezultati rasejanja polja u slučaju PEC cilindra i slojevite sredine sa gubicima.
\end{abstract}

\section{Key words:}

FDTD metoda,

TF/SF - Total Field/Scattered Field.

\section{UVOD}

U FDTD (Finite Difference Time Domain) računarskim simulacijama elektromagnetskih polja koriste se različiti tipovi eksitacija. Izbor eksitacije se vrši na osnovu više faktora: dimenzije FDTD domena (1D, 2D, 3D prostor), geometrije numeričkog domena izračunavanja (talasovod, optički kabl, itd.), fizičke strukture prostora (slobodan prostor, poluprostor, PEC (Perfect electric conductor) sloj). Najčešće se koriste tačkasti izvori, čija su polja zasnovana na Gausovoj, Rikerovoj ili sinusoidnoj funkciji. Karakteristika tačkastih izvora je da se njihova polja prostiru u svim pravcima usvojenog FDTD numeričkog domena. Međutim, postoje problemi polja kada je neophodno u FDTD simulaciju implementirati izvor čije se polje prostire isključivo u jednom pravcu. Pokazano je da je jedan od najefikasnijih načina da se to postigne ako se koristi TF/SF (Total Field/Scattered Field) tehnika eksitacije [1].

TF/SF tehnika omogućava FDTD modelovanje dugotrajnih ili kratkotrajnih impulsnih ili sinusoidnih eksita- kom pravcu u izabranom numeričkom domenu. Mnogi problemi elektromagnetskih polja, kao što su izračunavanje površine radarskog preseka (Radar cross-section), kao i analiza polja antena u dalekoj zoni, mogu se rešavati koristeći ovu tehniku [2]. TF/SF tehnika je prvi put uvedena u [3], polazeći od Hajgensovih površina (Huygen's surfaces), dok je detaljna diskusija i implementacija TF/SF ravnih talasa data u [1].

Istorijski gledano, TF/SF tehnika je razvijena kako bi se omogućilo generisanje ravnih talasa u FDTD metodi, ali u teoriji je, pomoću ove tehnike, moguće generisati bilo koji tip incidentnog talasa.

U ovom radu su razmatrani isključivo ravni talasi koji se prostiru samo u jednom pravcu, pri čemu je prvo opisan osnovni princip na kom je zasnovana TF/SF tehnika, a zatim i način njene implementacije $u$ računarsku FDTD simulaciju. Za dve karakteristične 2D geometrije, slučaj slobodnog prostora sa PEC cilindrom i slučaj dva homogena dielektrična poluprostora (slobodan prostor i sloj sa gubicima), urađene su 2D FDTD simulacije elektromagnetskih polja i priloženi su rezultati primene tehnike TF/ SF ravnih talasa, koji u osnovi imaju sinusoidni i Rikerov impuls. 


\section{TF/SF TEHNIKA}

Formulacija TF/SF tehnike bazirana je na osobinama linearnosti Maksvelovih jednačina i na superpoziciji komponenti totalnog električnog i magnetnog polja $\mathrm{E}_{\text {tot }} \mathrm{i}_{\text {tot }}$ :

$$
\mathrm{E}_{\text {tot }}=\mathrm{E}_{\text {inc }}+\mathrm{E}_{\text {scat }}, \mathrm{H}_{\text {tot }}=\mathrm{H}_{\text {inc }}+\mathrm{H}_{\text {scat }},
$$

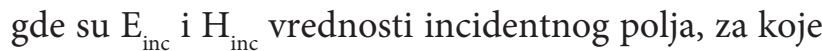
se pretpostavlja da je poznato u svim tačkama prostorne FDTD matrice, $i$ to $\mathrm{u}$ svakom vremenskom koraku, a $\mathrm{E}_{\mathrm{s}}$ ${ }_{\text {cat }} \mathrm{i}_{\text {scat }}$ su vrednosti rasejanog (skaterovanog) polja, čije vrednosti, inicijalno, nisu poznate. FDTD jednačine Yeeovog algoritma [4] su primenjive na incidentno, rasejano i totalno polje.

Na sl. 1 prikazan je numerički FDTD domen sa TF/ SF granicom (boundary), na kojoj se vidi kako je Yee-ova rešetka podeljena na dve oblasti: TF region (oblast komponenti totalnog polja) i SF region (oblast komponenti reflektovanog polja). TF/SF granica je virtuelna površina, pravougaonog oblika, koja povezuje ove dve oblasti i u njoj se generiše incidentni talas. Lokacija ove granice je donekle proizvoljna, ali je obično pozicionirana tako da rasejač, ukoliko ga ima, bude u TF regionu.

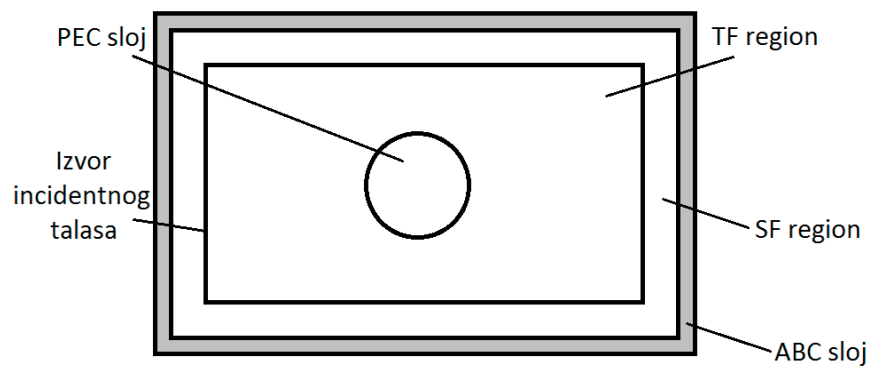

S. 1. Ilustracija FDTD domena sa TF/SF granicom i PEC cilindrom

U TF regionu (oblasti), koji obuhvata unutrašnji deo FDTD domena, Yee-ov algoritam operiše sa komponentama totalnog polja, koje su suma komponenti incidentnog i reflektovanog polja. U TF regionu se, uglavnom, nalaze PEC slojevi, ili slojevi sa gubicima.

U SF regionu (oblasti), koji obuhvata spoljni deo FDTD domena, Yee-ov algoritam operiše samo sa komponentama reflektovanog polja, što znači da u ovom regionu nema komponenti incidentnog polja. Oko SF regiona, nalazi se ABC (Absorbing Boundary Condition) sloj, sloj kojim je zatvoren (završen) FDTD domen.

$\mathrm{Na}$ TF/SF granici koriguju se vrednosti komponenti polja u okolini granice, tj. menjaju se vrednosti polja čije se susedne zavisne komponente (zavisne u smislu Yeeovog algoritma) nalaze sa druge strane granice.

U TF regionu, u području odmah pored TF/SF granice, nedostaju komponente incidentnog polja, koje bi prostorno trebalo da se nalaze u SF regionu. U SF regionu ne postoje komponente incidentnog polja, te da bi Yee-ov algoritam ostao konzistentan, potrebno je u ovim tačkama FDTD domena dodavati komponente incidentnog polja koje nedostaju.
S druge strane, u SF regionu, komponentama reflektovanog polja, lociranim pored TF/SF granice, neophodno je oduzimati komponente incidentnog polja, kako bi Yeeov algoritam ostao konzistentan. Detaljno objašnjenje i implementacija TF/SF granice u FDTD algoritam za 1D, 2D i 3D slučajeve dato je u [1].

Jedna od prednosti TF/SF tehnike je mogućnost generisanja proizvoljnog tipa talasa. Naime, uslovi pod kojima su na TF/SF granici povezani TF i SF regioni, omogućavaju generisanje talasa proizvoljnog talasnog oblika, trajanja, ugla incidencije i tipa polarizacije. Granica osigurava da se incidenti talas unutar TF regiona prostire samo u željenom pravcu. Relativno prosto implementiranje interakcionih struktura u FDTD numerički domen proističe iz činjenice da se različiti materijali, strukture i rasejači nalaze isključivo u TF regionu.

Dobra strana TF/SF tehnike je i veoma širok dinamički numerički opseg, posebno u slučajevima kada je potrebno precizno izračunati elektromagnetsko polje u „skrivenim“ delovima FDTD domena, gde su komponente polja za red veličine manje od jačine polja incidentnog talasa. Jasna razdvojenost SF regiona od TF regiona, tj. jasna razdvojenost komponenti incidentnog polja od komponenti reflektovanog polja, omogućava laku i efikasnu implementaciju $\mathrm{ABC}$ graničnih uslova.

\section{PRIMENA TF/SF TEHNIKE U 2D FDTD SIMULACIJAMA}

Da bi se ocenila implementacija TF/SF ravnih talasa, koji u osnovi imaju sinusoidni i Rikerov impuls, napisan je FDTD programski kod u $C++$ programskom jeziku. Korišćen je standardni eksplicitni algoritam FDTD metode. Rezultati numeričkih FDTD simulacija polja predstavljeni su pomoću grafičkog programa gnuplot. Poznato je da je u slučaju FDTD simulacija polja realnih fotoničkih i mikrotalasnih struktura potrebno izvršiti bar nekoliko hiljada vremenskih koraka. Međutim, prednosti korišćenja TF/SF tehnike se mogu uočiti već posle nekoliko stotina vremenskih koraka u FDTD simulaciji, što se jasno može videti iz priloženih rezultata $\mathrm{u}$ ovom radu. U podsekciji $A$. predstavljeni su rezultati 2D FDTD simulacija rasejanja polja u obliku TF/SF ravnog talasa, koji u osnovi ima Rikerov impuls i prostire se u slobodnom prostoru sa PEC cilindrom. $U$ podsekciji $B$. prikazani su rezultati $2 \mathrm{D}$ FDTD simulacija rasejanja polja u obliku TF/SF ravnog talasa, koji u osnovi ima sinusoidni impuls i prostire se u nehomogenoj sredini sastavljenoj od dva dielektrična poluprostora: slobodnog prostora i sloja sa gubicima.

\section{TF/SF Rikerov impuls u slobodnom prostoru sa PEC cilindrom}

U ovoj 2D FDTD simulaciji elektromagnetsko polje je eksitovano u obliku TF/SF ravnog talasa koji u osnovi ima Rikerov impuls. Talas se prostire u slobodnom prostoru. Rasejač u obliku PEC cilindra poluprečnika 35 prostornih ćelija, smešten je u centru numeričkog FDTD domena. Cilj simulacije je da prikaže prednost primene TF/SF ravnog talasa u FDTD simulacijama u kojima se zahteva 
ravnomerna iluminacija nekog PEC sloja, elektromagnetskim poljem iz jednog pravca. Prostiran je TM tip talasa, a funkcija izvora je [7]:

$$
f_{r}(q)=\left(1-2 \pi^{2}\left[\frac{C_{n} q}{N_{P}}-\mathrm{M}\right]^{2}\right) e^{-\pi^{2}\left[\frac{C_{n} q}{N_{P}}-\mathrm{M}\right]^{2}},
$$

gde je $C_{n}=c \Delta t / \Delta x$ - Kuronov broj (Courant number), kojim se definiše numerička stabilnost FDTD algoritma, $\Delta t$ predstavlja vremenski, $\Delta x$ prostorni korak, dok je $c$ brzina prostiranja elektromagnetskih talasa u slobodnom prostoru, $q$ je vreme, $N_{p}=\lambda_{p} / \Delta x$ - broj prostornih koraka po talasnoj dužini $\lambda_{P}$, kojim se definiše širina impulsa. $M$ je proizvoljna konstanta koja utiče na vremensko kašnjenje, i u simulaciji, sprovedenoj u ovom radu, $M=1$.

Širina Rikerovog impulsa je $N_{P}=30$. Za FDTD prostorni 2D domen usvojen je format od $x=200$ i $y=200$ ćelija, a ukupno trajanje simulacije je ograničeno na 400 vremenskih koraka. TF region se prostire od 20. do 180 . prostorne ćelije u pravcu $x$ ose i od 20. do 180. u pravcu $y$ ose. SF region obuhvata ostatak FDTD domena, tj. od 181. do 200. prostorne ćelije, u oba pravca. Numerički FDTD domen je ograničen Murovim graničnim uslovima drugog reda [6]. TF/SF ravni talas sa Rikerovim impulsom pobuđen je u TF/SF granici, duž leve ivice domena.

Na sl. 2, sl. 3, sl. 4 i sl. 5 prikazani su intenziteti $E_{z}$ komponente rasejanog polja u transverzalnoj ravni, TM tip talasa, posle 80, 160, 240 i 300 vremenskih koraka FDTD simulacije, eksitacije u obliku TF/SF ravnog talasa koji u osnovi ima Rikerov impuls, a prostire se u slobodnom prostoru i nailazi na PEC sloj u obliku cilindra.

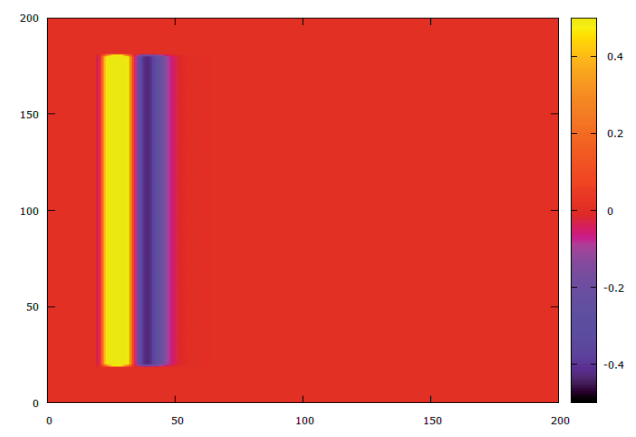

Sl. 2 Intenzitet $E_{z}$ komponente polja u transverzalnoj ravni pri TS/SF eksitaciji Rikerovim impulsom posle 80 vremenskih koraka

S1. 2 prikazuje polje u trenutku u kom je generisan talas. Uočljivo je da je incidentni talas ograničen samo na TF region i da se prostire samo u pravcu $x$ ose. Takođe se vidi da nema nikakvih drugih (neželjenih!) komponenti koje se prostiru u nekom drugom pravcu.

Sl. 3, sl. 4 i sl. 5 ilustruju refleksiju talasa od PEC cilindra. Može se primetiti da se komponente reflektovanog talasa prostiru kroz SF region, dok komponente incidentnog talasa ostaju u TF regionu do kraja FDTD simulacije.

\section{TF/SF sinusoidalni impuls u nehomogenoj sredini}

U ovoj 2D FDTD simulaciji elektromagnetsko polje je eksitovano u obliku TF/SF ravnog talasa koji u osnovi ima sinusoidalni impuls. Talas je prostiran u nehomogenoj sredini sastavljenoj iz dva homogena dielektrična poluprostora. Cilj simulacije je da prikaže prednost primene kontinuiranih TF/SF ravnih talasa u FDTD simulacijama u kojima se zahteva ravnomerna iluminacija nekog PEC sloja, EM poljem iz jednog pravca. Prostiran je TM tip talasa, a funkcija izvora je [7]:

$$
f_{r}(q)=\sin \left(\frac{2 \pi}{N_{P}}\left[C_{n} q-\mathrm{M}\right]\right) .
$$

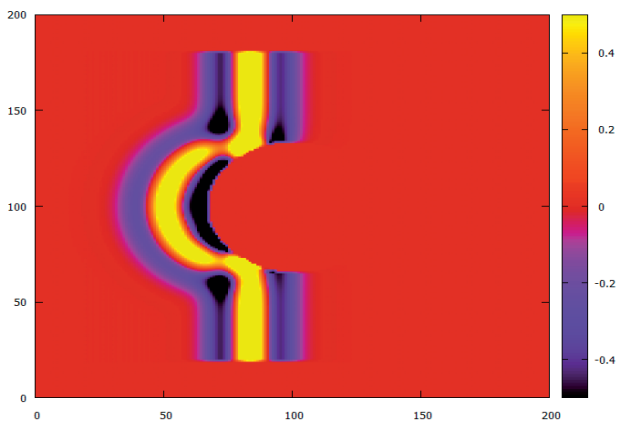

Sl. 3. Intenzitet $E_{z}$ komponente polja u transverzalnoj ravni pri TS/SF eksitaciji Rikerovim impulsom posle 160 vremenskih koraka

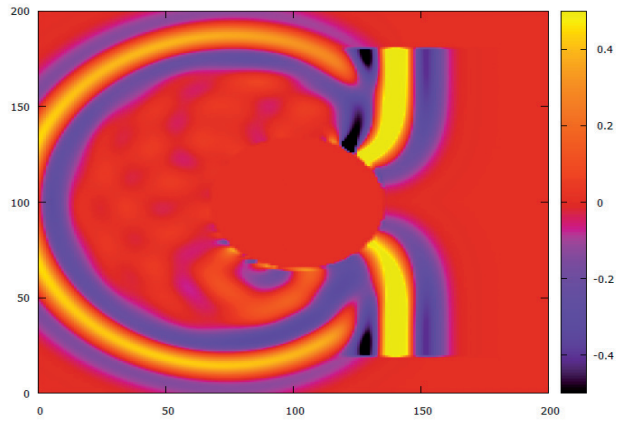

Sl. 4. Intenzitet $E_{z}$ komponente polja u transverzalnoj ravni pri TS/SF eksitaciji Rikerovim impulsom posle 240 vremenskih koraka

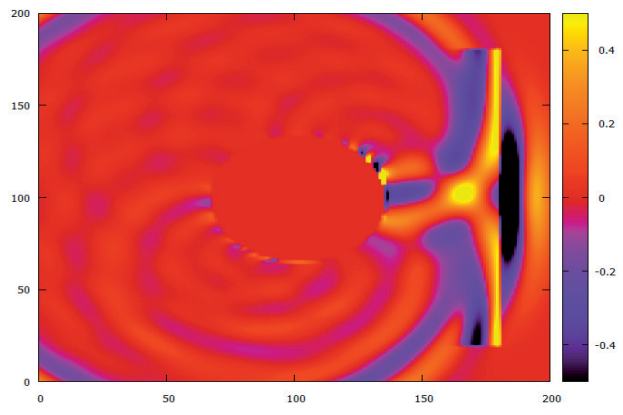

S. 5. Intenzitet $E_{z}$ komponente polja u transverzalnoj ravni pri TS/SF eksitaciji Rikerovim impulsom posle 300 vremenskih koraka

Širina sinusoidnog impulsa je $N_{P}=30$. Dimenzija FDTD domena, kao i trajanje simulacije isto je kao $\mathrm{u}$ podsekciji $A$. Dimenzije TF i SF regiona su, takođe, iste, 
kao i granični uslovi na granicama domena. Slobodan poluprostor se nalazi u TF regionu i prostire se od 20. do 100. prostorne ćelije, a od 101. do 180. je poluprostor s gubicima. Konstanta slabljenja u poluprostoru s gubicima je $\alpha=0.0253146$, relativna permitivnost je $\varepsilon_{r}=4$, a relativna permeabilnost je $\mu_{r}=1$.

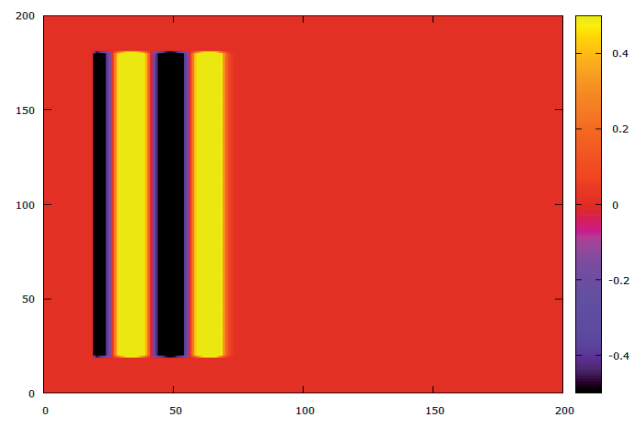

Sl. 6. Intenzitet $E_{z}$ komponente polja u transverzalnoj ravni pri TS/SF eksitaciji sinusoidalnim impulsom posle 100 vremenskih koraka

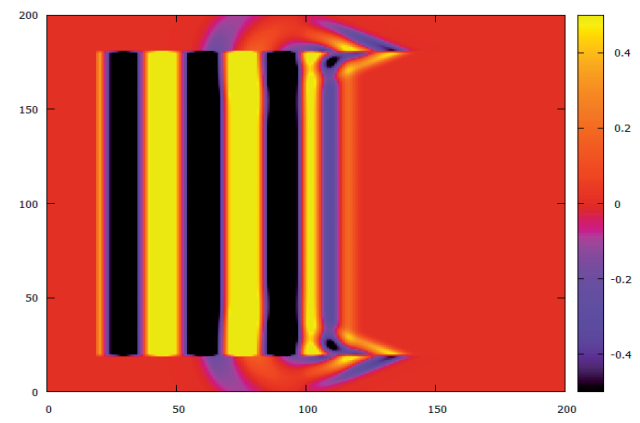

Sl. 7. Intenzitet $E_{z}$ komponente polja u transverzalnoj ravni pri TS/SF eksitaciji sinusoidalnim impulsom posle 200 vremenskih koraka

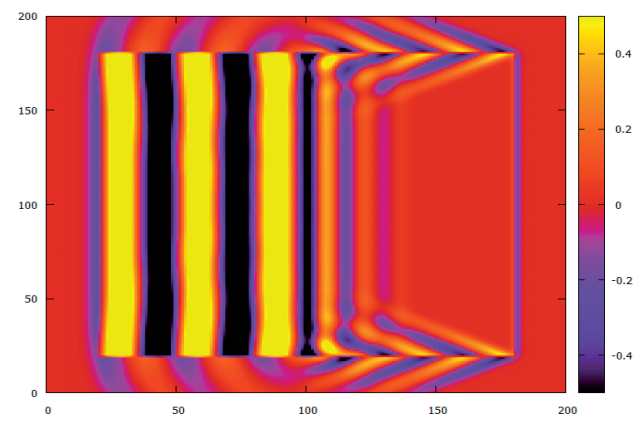

Sl. 8. Intenzitet $E_{z}$ komponente polja u transverzalnoj ravni pri TS/SF eksitaciji sinusoidalnim impulsom posle 260 vremenskih koraka

Na sl. 6, sl. 7, sl. 8 i sl. 9 prikazani su intenziteti $E_{z}$ komponente rasejanog polja u transverzalnoj ravni, TM tip talasa, posle 100, 200, 260 i 300 vremenskih koraka FDTD simulacije, eksitacije u obliku TF/SF ravnog talasa koji u osnovi ima sinusoidni impuls, u nehomogenoj sredini sastavljenoj iz dva dielektrična poluprostora.

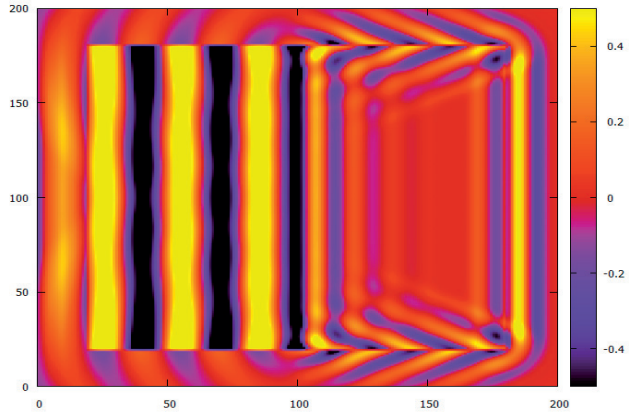

Sl. 9. Intenzitet $E_{z}$ komponente polja u transverzalnoj ravni pri TS/SF eksitaciji Rikerovim impulsom posle 300 vremenskih koraka

Sl. 6 prikazuje trenutak generisanja talasa, gde se vidi da nema neželjenih komponenti talasa u pravcu suprotnom od željenog pravca prostiranja. Sl. 7, sl. 8 i sl. 9 prikazuju kako talasa slabi, nakon prostiranja kroz sloj sa gubicima. I u ovom slučaju komponente incidentnog talasa su ograničene na TF region, a kroz SF region se prostiru isključivo komponente reflektovanog talasa.

\section{ZAKLJUČAK}

Predstavljeni su rezultati 2D FDTD simulacija rasejanja polja pobuđenog u obliku TF/SF ravnog talasa, koji u osnovi ima Rikerov i sinusoidni impuls, za slučaj rasejača 1) u obliku poprečno postavljenog PEC cilindra i 2) granične površi dva homogena dielektrična poluprostora. Rezultati FDTD simulacija pokazuju efikasnost izbora eksitacije u obliku TF/SF ravnog talasa u slučaju analiza polja koja se prostiru u jednom pravcu.

\section{LITERATURA}

[1] A.Taflove and S.C.Hagness, Computational Electrodynamics: The Finite-Difference Time-Domain Method, 3rd ed, Artech House, 2005.

[2] Dennis M. Sullivan, Electromagnetic Simulation Using the FDTD Method, IEEE press, 2000.

[3] Merewether, D. E., R. Fisher, and F. W. Smith. "On implementing a numeric Huygen's source scheme in a finite difference program to illuminate scattering bodies.", IEEE Transactions, Nuclear Science, vol. 27, no. 6, pp. 1829-1833, Dec. 1980.

[4] Yee, Kane, "Numerical solution of initial boundary value problems involving Maxwell's equations in isotropic media", Antennas and Propagation, IEEE Transactions,vol.14, issue 3, pp. 302-307, May 1966.

[5] Schneider, John B. "Plane waves in FDTD simulations and a nearly perfect total-field/scattered-field boundary." IEEE Transaction, Antennas and Propagation, vol. 52, no. 12, pp. 3280-3287, Dec. 2004.

[6] Mur, Gerrit, "Absorbing boundary conditions for the finite-difference approximation of the time-domain electromagnetic-field equations", Electromagnetic Compatibility, IEEE Transactions on, vol. 23, issue 4, pp. 377-382, 1981. 
[7] John B. Schneider, Understanding the Finite-Difference Time-Domain Method, www.eecs.wsu.edu/ sschneidj/ ufdtd, 2010.

\section{APPLICATION OF TF/SF EXCITATION IN FDTD COMPUTER SIMULATIONS OF ELECTROMAGNETIC FIELDS}

Abstract:

In FDTD (Finite Difference Time Domain) computer simulations of electromagnetic fields there is often a necessity for sources which field propagates in only one direction of the adopted computational domain, beside point sources which field propagate in all directions. TF/SF (Total Field/Scattered Field) plane waves are efficient choice for field/wave propagation in one defined direction. Basic concept and FDTD implementation of TF/SF plane waves are presented in this paper. The numerical results obtained by 2D FDTD computer simulations performed in the case of TF/SF waves with Ricker and sinusoidal source functions for PEC cylinder in free space and for non-homogeneous media are given and discussed.

Key words:

FDTD method,

TF/SF - Total Field/Scattered Field; 